\title{
Treatment Outcomes of Tuberculosis Patients at Debre Berhan Hospital, Amhara Region, Northern Ethiopia
}

\author{
FirdieTefera $^{1,}$ Tariku Dejene ${ }^{2,}$ Tsegaye Tewelde ${ }^{3}$
}

\begin{abstract}
BACKGROUND: Directly observed treatment remains one of the most widely-accepted global health interventions for tuberculosis. Tuberculosis treatment outcome is one of the performance indicators of the programme set by World Health Organization. Therefore, evaluating the treatment success rate ofDebreBerhanHospital was mandatory to show the achievement and to indicate where the hospital is against the World Health Organization target.

METHODS: A register based cross sectional study covering the period of January 2009 to December 2013 was employed. All clients with complete records of the treatment outcome were included in the study. A checklist was prepared to extract data from patient charts. Data were entered into Epi-info version 3.2.2andanalyzed using SPSS version 16 for windows.

RESULT: Between January 2009 and December 2013, a total of 1280 tuberculosis cases that had complete records on treatment outcome were included in the analysis. Four in five (79.4\%) of the patients had favorable treatment outcome; $15.8 \%$ were cured and $63.5 \%$ completed their treatment. There was a continuous increment of treatment success rate from 2010 to 2013 in the area and the treatment success rate in the year 2013 was $84.4 \%$.

CONCLUSION: Despite the recent improvements in treatment success rate, treatment completed and defaulting rate, further efforts should be made by responsible bodies to identify and improve possible promoting factors for successful tuberculosis treatment outcome.

KEYWORDS: Tuberculosis, Treatment outcome, Success rate, DOTs, DebreBerhan
\end{abstract}

DOI:http://dx.doi.org/10.4314/ejhs.v26i1.11

\section{INTRODUCTION}

Tuberculosis is an infectious disease caused by Bacillus Mycobacterium Tuberculosis. The disease mainly affects the lung (pulmonary tuberculosis). However, it can also affect other parts of human body (extra pulmonary tuberculosis) (1).

Directly Observed Treatment (DOTS) remains one of the most widely-accepted global health interventions for tuberculosis. The targets of DOTs are to achieve a case detection rate (CDR) of $84 \%$ (of all forms of TB) and treatment success rates (TSR) of $87 \%$ by $2015(1)$. With regard to the burden of tuberculosis, Ethiopia is among the top sixteen countries in the world and the three countries in Africa (2). And, it is also the leading cause of morbidity, the third cause of hospital admission and the second cause of death in Ethiopia (2).

Treatment outcome and detection rate are the performance indicators of DOTs programme set by World Health Organization $(\mathrm{WHO})(3,4)$ of which reaching a treatment success rate of $87 \%$ by 2015 waslaunched as a target by $\mathrm{WHO}(3)$.Disease surveillance system and treatment outcome monitoring are considered as the key source of

\footnotetext{
${ }^{1}$ Zonal Health Office, North Shoa, Ethiopia

${ }^{2}$ Center for Population Studies, College of Development Studies, Addis Ababa University, Ethiopia

${ }^{3}$ Department of Epidemiology and Biostatistics, Jimma University, Jimma, Ethiopia

Corresponding Author: TsegayeTewelde: tsegaye.tewelde@yahoo.com
} 
evidence to evaluate the effectiveness of tuberculosis prevention and control programmes like DOTs (5).

In Ethiopia, Directly Observed Treatment, short Course (DOTs) was introduced in 1992 as a pilot, and further scaled up at national level. Though, the health facility coverage is $75 \%$ in Ethiopia, DOTs reach coverage of $90 \%(6,7)$. DOTs given for at least the first two months of treatment, under the direct observation of health care providers has been found to be effective in achieving a highly successful treatment outcome ranging from 86 to $96.5 \%$ (8). Similarly, treatment success rate is considered as a proxy indicator of the quality of health care services, and it is usually affected by various reasons (6).

Despite the existence of increment in treatment success rate in almost all of the studies done in Ethiopia (4, 6, 9-11), the average treatment success rate in most of them was below the MDG target (3). And,almost all were conducted before the year 2009. On top of these, we are left with almost a year for the 2015 MDG tuberculosis performance indicator target. Therefore, evaluating the treatment success rate of TB in DebreBerhanHospital is mandatory to show the achievement and to indicate where the hospital is against the WHO target by 2015.Thus, the aim of this study was todetermine treatment success rate of tuberculosis patients under antituberculosis treatment from January, 2009 to December 2013 in DebreBerhanHospital. Determining the treatment success rate of the hospital will help health planners and health professionals to show where they are relative to MDG tuberculosis target. In case if the TSR is under the target, it could lead them to identifying potential areas of improvement. This in turn could have its own role in preventing multidrug resistance (MDR-TB) and Xetensive drug resistance (XDR-TB) tuberculosis.

\section{METHODS AND PARTICIPANTS}

Study setting and design: Secondary data were collected from April to May 2014 from a registration book of TB patients who had been treated from January 2009 to December 2013 in DebreBerhanHospital, which is one of the four governmental hospitals in North Shewa Zone, Amhara Regional State, Ethiopia. DebreBerhanHospital is performing TB diagnosis, treatment and other related activities (VCT, prevention of mother to child transmission (PMTCT), antiretroviral therapy (ART) and opportunistic infections treatment services). A retrospective document review cross sectional study design was employed.

Participants: All eligible TB patients who took anti-TB treatment from January 2009 to December 2013 in DebreBerhanHospital were included in the study. Eligibility was based on completeness of record on treatment outcomes of a patient in the TB registration book and period of enrollment to DOTs treatment.

Measurement: Tuberculosis treatment outcome was dichotomized as treatment success (cure or treatment completed as indicated in the unit TB register) or poor treatment outcome (treatment failure or death or defaulter or transferred out as indicated in the unit TB register). 'Cured' was defined as patients whose sputum smear or culture was positive at the beginning of the treatment but smear or culture negative in the last month of treatment and on at least one previous occasion. Similarly, 'treatmentcompleted'was interpreted as patients who completed treatment without evidence of failure but did not have a negative sputum smear or culture result in the last month of treatment and on at least one previous occasion. 'Treatmentdefaulter' defined as was a patient who had been on treatment for at least four weeks and whose treatment was interrupted for eight or more consecutive weeks. 'Treatment failure' was defined as patients whose sputum smear or culture was positive at the fifthmonthor later during treatment or patients found to harbor a multidrugresistant (MDR) strain at any point of time during the treatment whether they are smear negative or positive. Similarly, 'transfer out' was defined as a patient who had been transferred to another recording and reporting unit and whose treatment outcome was unknown at the original registering unit.

Other variables included in the study were socio-demographic characteristics (age, sex, weight and residence), disease and services related factors (provider-initiated testing and counseling (PITC), HIV test result, ART, type of tuberculosis (PTB+, PTB-, and EPTB), category of TB patients (new, relapse, treatment after failure, return after default, transfer in), year of enrolment, 
followupsputum smear result at the second month, fifth month, sixth month, eight month.

TB patients' records review were made for data extraction from the registration of the TB Clinic of the Hospital. Two nurses working at the TB slinicsin other health facilities and one supervisor who was TB officer in another district health office were recruited and trained for data extraction. The extraction of variables like demographic, disease related, health services related and treatment outcomes was undertaken using a checklist developed by the investigators.

Sampling procedure: The sample size was calculated using single population proportion formula, by taking estimated average tuberculosis treatment success rate of $54 \%$ because treatment success rate ranges from 29.5-82.7\% (2, 4, 6, 1012) in Ethiopia. Considering $95 \%$ Confidence level, 5\% margin of error and finally with the addition of $15 \%$ non-response (incomplete records for treatment outcome) a total of 449tuberculosis patient recordswere required. However, since all the data were extracted from records and to increase the precision of the study, all TB patients of DebreBerhanHospital who started DOTs and had complete medical records on treatment outcome in the past five years (January 2009 to December 2013) preceding the data collection period were included in the study.

Data processing and analysis: Data were edited manually before entry to a computer and then entered to Epi-info version 3.2.2. Thereafter, they were cleaned, coded and analyzed using SPSS version 16 for windows. A descriptive analysis was conducted to get summary values of treatment outcome of TB and other explanatory variables as well as to check for outliers, inconsistencies and missed values. Finally, the results were presented in the form of tables and charts.
Ethical consideration: Ethical clearance was obtained from the Health Research and Postgraduate Coordinating Office of the College of Public Health and Medical Sciences of Jimma University. Support letter was obtained from Jimma University to North Shewa Zone Health Department. Then, the Zonal Health Office wrote a letter of support to DebreBerhanReferralHospital. To ensure confidentiality of the information, names of TB patients were not included in the checklist and data were used only for research purpose.

\section{RESULT}

Demographic and disease related characteristics: Between January 2009 and December 2013, a total of 1830 tuberculosis patients were registered in the TB register unit of the hospital. Of these, $1280(70 \%)$ tuberculosis cases had complete records on the treatment outcome. Among the total registered TB patients with complete record $(n=1280), 649(50.7 \%)$ were males and the median age of patients was 28 years. Near to one in ten of the study participants were children aged less than 15 years; near to seven in ten were adults with ages ranging from 15 through 44 years. About $72 \%(n=930)$ of the patients were urban residents. Three in ten (29.6\%) were smear positive PTB, nearly three fifth $(56.6 \%)$ were smear negative PTB and the rest were EPTB patients. 1190(93\%), 33 (2.6\%), $22(1.7 \%)$ and $35((2.7 \%)$ of TB patients were new, retreatment, transfer in and other cases, respectively. Seven in ten $(70.9 \%)$ were offered provider-initiated testing and counseling for HIV, of whom seven in ten $(71.1 \%)$ were HIV negative cases (Table1). 
Table 1: Socio-demographic and disease related characteristics of registered TB patients $(n=1280)$ in DebreBerhan referral Hospital, Jan. 2009 to Dec. 2013.

\begin{tabular}{|c|c|c|c|}
\hline Variable & Categories & Frequency & Percentage (\%) \\
\hline \multirow[t]{2}{*}{ sex } & Male & 649 & 50.7 \\
\hline & Female & 631 & 49.3 \\
\hline \multirow[t]{7}{*}{ Age in years } & $0-14$ & 141 & 11.0 \\
\hline & $15-24$ & 351 & 27.4 \\
\hline & $25-34$ & 341 & 26.6 \\
\hline & $35-44$ & 215 & 16.9 \\
\hline & $45-54$ & 117 & 9.1 \\
\hline & $55-64$ & 68 & 5.3 \\
\hline & $\geq 65$ & 47 & 3.7 \\
\hline \multirow[t]{2}{*}{ Residence } & Urban & 930 & 72.7 \\
\hline & Rural & 350 & 27.3 \\
\hline \multirow[t]{3}{*}{ Type of TB } & PTB+ & 379 & 29.6 \\
\hline & PTB- & 724 & 56.6 \\
\hline & ЕРТВ & 177 & 13.8 \\
\hline Category & New TB cases & 1190 & 93.0 \\
\hline \multirow[t]{5}{*}{ of TB patients } & Relapse cases & 26 & 2.0 \\
\hline & Treatment after failure & 2 & 0.2 \\
\hline & Return after default & 5 & 0.4 \\
\hline & Transfer in & 22 & 1.7 \\
\hline & Other & 35 & 2.7 \\
\hline \multicolumn{4}{|c|}{ Provider initiated testing and counseling offered } \\
\hline & Yes & 907 & 70.9 \\
\hline & No & 373 & 29.1 \\
\hline \multirow[t]{2}{*}{ HIV test result } & Positive & 262 & 28.9 \\
\hline & Negative & 645 & 71.1 \\
\hline
\end{tabular}

Treatment Outcomes: From the total of 1280 registered tuberculosis patients included in the study, 1016(79.4\%) had successful treatment outcome (favorable treatment outcome). Of these, 203(20\%) and 813(80\%) patients were cured and treatment completed, respectively. Similarly,135(10.5\%), 102(8\%), 23 (1.8\%) and $4(0.3 \%)$ were transferred out, defaulted, dead and treatment failed, respectively (Figure 1).

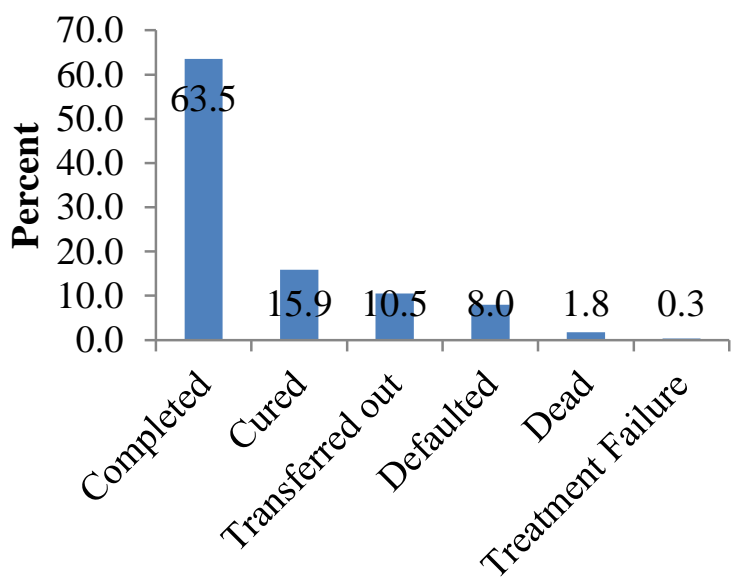

Figure 1: Treatment outcomes of patients on DOTS treatment $(n=1280)$ in DebreBerhan hospital from Jan. 2009 to Dec. 2013, Ethiopia 
Favorable treatment outcome slightly decreased from the year 2009 to 2010 (78.5 to $75.9 \%)$ whereas starting from the end of 2010 to 2013, there was a continuous increment on favorable treatment outcome of TB (figure 2). Patients who completed their treatment slightly increased from $58.5 \%$ in 2009 to $60.1 \%$ in 2010 , but there was a decline to $56.4 \%$ in 2011. Later, treatment completed continuously rose from $56.4 \%$ in 2011 to $75.3 \%$ in 2013. Similarly, the number of patients who were cured from tuberculosis decreased from $20 \%$ in 2009 to $15.8 \%$ in 2010 but again inclined to $20 \%$ in 2011. However, since then till 2013, a continuous decline was observed on cure rate. On top of these, treatment defaulting continuously increased from $6.9 \%$ in 2009 to $9.4 \%$ in 2012 but finally decreased to $5.7 \%$ in 2013(Figure 2).

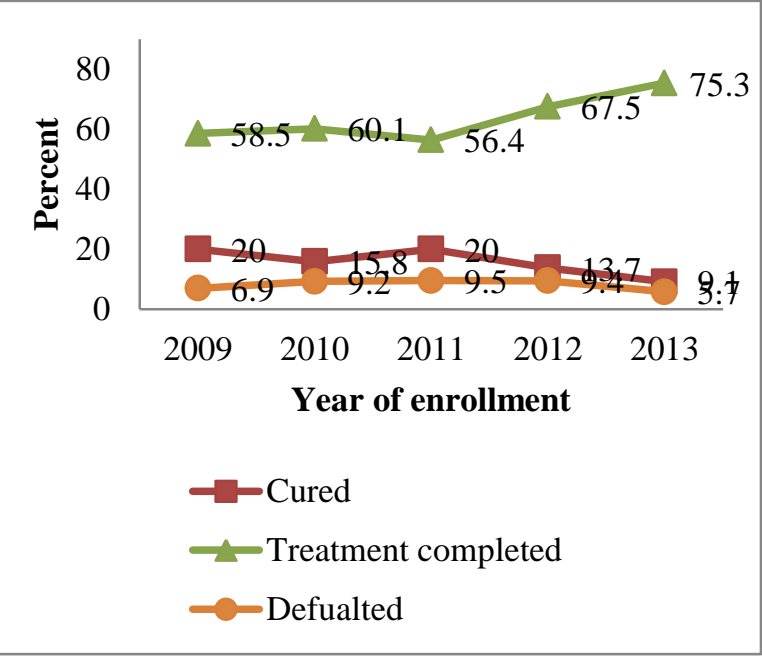

Figure 2: Trends of treatment outcomes of patients on DOTS treatment $(n=1280)$ in DebreBerhan hospital from Jan. 2009 to Dec. 2013, Ethiopia.

Table 2: Distribution of treatment outcomes of registered TB patients $(n=1280)$ by socio-demographic and disease characteristics in DebreBerhanHospital, Jan. 2009 to Dec. 2013.

\begin{tabular}{|c|c|c|c|c|}
\hline \multirow[b]{2}{*}{ Variables } & \multirow[b]{2}{*}{ Categories } & \multicolumn{2}{|c|}{ Treatment outcome } & \multirow[b]{2}{*}{ Total n $(\%)$} \\
\hline & & $\begin{array}{l}\text { Favorable } \\
\text { outcome } \mathrm{N}(\%)\end{array}$ & $\begin{array}{l}\text { Unfavorable outcome } \\
\mathrm{N}(\%)\end{array}$ & \\
\hline Sex & Male & $511(78.7)$ & $138(21.3)$ & $649(100)$ \\
\hline Residence of patients & $\begin{array}{l}\text { Female } \\
\text { Urban }\end{array}$ & $\begin{array}{l}505(80) \\
753(81)\end{array}$ & $\begin{array}{l}126(20) \\
177(19)\end{array}$ & $\begin{array}{l}631(100) \\
930(100)\end{array}$ \\
\hline \multirow[t]{6}{*}{ Age category } & $\begin{array}{l}\text { Rural } \\
0-14\end{array}$ & $\begin{array}{l}263(75.1) \\
108(76.6)\end{array}$ & $\begin{array}{l}87(24.9) \\
33(23.4)\end{array}$ & $\begin{array}{l}350(100) \\
141(100)\end{array}$ \\
\hline & $15-24$ & $278(79.2)$ & $73(20.8)$ & $351(100)$ \\
\hline & $25-34$ & 277(81.2) & $64(18.8)$ & $341(100)$ \\
\hline & $35-44$ & 176(81.9) & $39(18.1)$ & $215(100)$ \\
\hline & $45-54$ & $91(77.8)$ & $26(22.2)$ & 117(100) \\
\hline & $55-64$ & $52(76.5)$ & $16(23.5)$ & $68(100)$ \\
\hline & $\begin{array}{l}\geq 65 \\
\text { New TB cases }\end{array}$ & $\begin{array}{l}34(72.3) \\
950(79.8)\end{array}$ & $\begin{array}{l}13(27.7) \\
240(20.2)\end{array}$ & $\begin{array}{l}47(100) \\
1190(100)\end{array}$ \\
\hline \multirow{4}{*}{ Category of TB patients } & Relapse Cases & $22(84.6)$ & $4(15.4)$ & $26(100)$ \\
\hline & $\begin{array}{l}\text { Treatment after } \\
\text { Failure }\end{array}$ & 0 & $2(100)$ & $2(100)$ \\
\hline & Return after default & $3(60)$ & $2(40)$ & $5(100)$ \\
\hline & Transfer in & $16(72.7)$ & $6(27.3)$ & $22(100)$ \\
\hline \multirow[t]{3}{*}{ Type of TB } & $\begin{array}{l}\text { Other } \\
\text { PTB+ }\end{array}$ & $\begin{array}{l}25(71.4) \\
309(81.5)\end{array}$ & $\begin{array}{l}10(28.6) \\
70(18.5)\end{array}$ & $\begin{array}{l}35(100) \\
379(100)\end{array}$ \\
\hline & PTB- & $567(78.3)$ & $157(21.7)$ & $724(100)$ \\
\hline & ЕРТВ & 140(79) & $37(21)$ & $177(100)$ \\
\hline
\end{tabular}


Distribution of treatment outcome by sociodemographic and disease characteristics: Five hundred eleven $(78.7 \%)$ of the patients with favorable outcome, and $138(21.3 \%)$ of the patients with unfavorable outcome were male. Similarly, near to eight in ten of the patients with favorable outcome were urban in residence, new tuberculosis cases, pulmonary positive tuberculosis cases and pulmonary negative tuberculosis cases respectively (Table 2).

\section{DISCUSSION}

In this study, the average treatment success rate of tuberculosis patients was found to be $79.4 \%$, and this was similar with an average of $80 \%$ in Southern Ethiopia (9),77\% between 2005 and 2011 in New Delhi, India (13). However, this was lower than the WHO international target of $87 \%$ by 2015 (3), 84\% in Southern State of India in 2011(14), 82.7\% in Addis Ababa, Ethiopia (4), $84.7 \%$ WHO report in 2005(15) and $94.8 \%$ in North West Ethiopia (12). This could be justified as population of Addis Ababa and India have better socio-demographic (educational) and economic condition than population in the catchment area of DebreBerhan in North Shewa Zone. This could also be as a result of difference in followup of patients and hence affects the outcome. On the contrary, the treatment success rate of this study was higher than an average of $49 \%$ in Hadiya Zone, Southern Ethiopia (10) and $55.7 \%$ in Gambella, Ethiopia (11). This could be justified as tuberculosis patients registered on the DOTs service between 2009 and 2013 were included in this study, which is relatively recent in time as compared to the previously mentioned studies. Hence, the quality of DOTs services and patients awarenessof the importance of TB treatment might have improved through time.

There was a continuous increment of treatment success rate from the end of 2010 to 2013 in the hospital. The treatment success rate in the year 2013 was $84.4 \%$, which is almost comparable with the WHO international target of $87 \%$ (3). This might be a clue indicating that the hospital is within the track of WHO target currently.

The average cure rate of tuberculosis was $15.9 \%$. This was consistent with an average cure rate of $18 \%$ in Addis Ababa, Ethiopia (4) and $18.7 \%$ in North West Ethiopia (12). However, this was lower than a finding from a study done in Gambella, Ethiopia (30.7\%) (11). This was because participants in the Gambella study were pulmonary TB (PTB+ and PTB-) cases only whereas both pulmonary and extra pulmonary tuberculosis cases were included in this study, which might underestimated the cure rate.

Similarly, the average treatment completed rate was $63.5 \%$ in this study, which was in agreement with $64.6 \%$ according to study done in Addis Ababa, Ethiopia (4). However, it was higher than: 9.5\% in Gondar Hospital, Northwest Ethiopia (6), and $25 \%$ in Gambella, Ethiopia (11). This variation might be due to difference in patients' enrollment time, patients registered in DOTs before the year 2009 were included in almost all of the previous studies unlike this study (in which patients registered between the year 2009 and 2013 were included).

As a result, the quality of DOTs services and patients' awareness on the importance of DOTs treatment might become better through time.

The average defaulting rate was $8 \%$, which was lower than an average of $11.3 \%$ in Arsi Zone, Ethiopia (16), $18.3 \%$ in Gondar Hospital, Northwest Ethiopia (6)and $18.6 \%$ in Hadiya, Southern Ethiopia (10). This could be because of differences in followup and counseling of patients in DOTs clinics across these settings, suitability of the institutional setup and patient demographic characteristics $(6,10,14,17)$, knowledge and attitude towards and side-effect due to DOTs (16)and due to difference in patient enrollment time as mentioned above. On top of these, differences in communities' beliefs on traditional healer or religious institutions for sick person might contribute to defaulting. However, the defaulting rate in this study was higher than $5.1 \%$ in a study done in Addis Ababa, Ethiopia (4). This might be due to differences in socio-demographic and economic status of more urbanized population in Addis Ababa as compared to the population in North Shewa Zone.

In addition, $1.8 \%$ of the TB patients died between 2009 and 2013, which was consistent with an average of $2.6 \%$ in Hadiya, Southern Ethiopia (10), 3.7\% in Addis Ababa, Ethiopia (4), and $3.4 \%$ in Northwest Ethiopia(12). However it was lower than $10.1 \%$ in Gondar Hospital, Northwest Ethiopia (6). The difference could be 
due to variation in HIV test offering status and anti-retroviral therapy initiation, participants in the study done in Gondar were TB patients who took DOTs from 2003 through 2008 in which the record did not show HIV test offer, test result and ART status (6)unlike in the current study ( near to $70.9 \%$ were offered HIV test counseling).Therefore, HIV positive TB cases can get anti-retroviral therapy and prevent mortality. On top of these, the average treatment failure rate was $0.3 \%$, consistent with $0.2 \%$ in Gondar Hospital, Northwest Ethiopia (6), 0.4\% in Addis Ababa, Ethiopia (4), and $0.5 \%$ in Northest Ethiopia (12).

Because of the large number of TB patients $(30 \%)$ with unknown treatment outcome due to incomplete record, there might have been under or over estimation of favorable treatment outcome of tuberculosis. Thus, care should be taken when utilizing the finding of this study. In conclusion, the average treatment success rate of tuberculosis patients registered in the DOTs programme was lower than the WHO international target, whereas the treatment success rate in the year 2013 was almost comparable with the WHO international target. This might be a clue indicating that the hospital is within the track of WHO target in 2015. Treatment completion rate increased unlike the cure rate and defaulting rate, which declined from 2011 through 2013. Similarly, the average treatment completion rate of patients was relatively higher than the rateidentifiedprevious studies in Ethiopia. And, the average defaulting and death rates of the area in the given years were almost lower than similar studies in Ethiopia, which indicated better success of the hospital.

There were improvements in the treatment success, defaulting and death rates. However, further effort should be done by responsible bodies to identify and improve possible promoting and hindering factors for successful tuberculosis treatment outcome.

\section{ACKNOWLEDGMENTS}

Our earnest gratitude goes to Jimma University for financial support on data collection phase of this research only, north Shewa Zone health office, DebreBerhan hospital, and study participants and data collectors for their valuable cooperation and assistance.

\section{REFERENCES}

1. Patrick OE, Winifred AO. Success of the Control of Tuberculosis in Nigeria: A Review. International Journal of Health Research, 2009; 2(1):3-14.

2. Gebretsadik B, Fikre E, Abraham A. Treatment outcome of smear-positive pulmonary tuberculosis patients in Tigray Region, Northern Ethiopia. BMC Public Health, 2012; 12(537):1471-2458.

3. World Health Organization: Global Plan to Stop TB 2011-2015. Davos, Switzerland: WHO;

2006.whqlibdoc.who.int/publications/2010/97 89241500340_eng.pdf, accessed febraury 2014.

4. Belete G, Gobena A, Girmay M, Sibhatu B. Treatment outcome of tuberculosis patients under directly observed treatment in Addis Ababa, Ethiopia. Braz J Infect Dis, 2013; 17(5):521-528.

5. World Health Organization: Global tuberculosis report 2014. Geneva 27, Switzerland: 2014.

WHO/HTM/TB/2014.08,www.who.int, accessed febraury 2014.

6. Belay T, Abebe M, Assegedech B, Dieter R, Frank E, Ulrich S. Treatment outcome of tuberculosis patients at Gondar University Teaching Hospital, Northwest Ethiopia: A five-year retrospective study. BMC Public Health, 2009; 9(371):1471-2458.

7. Ministry of Health of Ethiopia $(\mathrm{MOH})$ : Tuberculosis, Leprosy and TB/HIV Prevention and Control Programme Manual. Addis Ababa: 2008. 
8. Chaulk CP, Kazandjian VA: Directly observed therapy for treatment completion of pulmonary tuberculosis: Consensus Statement of the Public Health Tuberculosis Guidelines Panel. 1998; 280(2):134.

9. Yassin MA, Datiko DG, Shargie EB. Ten-year experiences of the tuberculosis control programme in the southern region of Ethiopia. Int J Tuberc Lung Dis, 2006; 10(10):11661171.

10. Shargie EB, Lindtjørn B. DOTS improves treatment outcomes and service coverage for tuberculosis in South Ethiopia: a retrospective trend analysis. BMC Public Health, 2005; $5: 62$.

11. Sisay S, Mengistu B, Erku W, Woldeyohannes D. Directly Observed Treatment Short-course (DOTS) for tuberculosis control program in Gambella Regional State, Ethiopia: ten years experience. BMC Res Notes, 2014; 7(44):1756-0500.

12. Endris M, Moges F, Belyhun Y, Woldehana E, Esmael A, Unakal C. Treatment outcome of tuberculosis patients at enfraz health center, northwest ethiopia: a five-year retrospective study. Tuberc Res Treat, 2014; 2014(726193):5.
13. Sharma SK, Soneja M, Prasad KT, Ranjan S. Clinical profile \& predictors of poor outcome of adult HIV-tuberculosis patients in a tertiary care centre in north India. Indian J Med Res, 2014; 139(1):154-160.

14. Ramya A, Kaliyaperumal K, Marimuthu G, et al. The profile and treatment outcomes of the older (aged 60 years and above) tuberculosis patients in Tamilnadu, South India. PLoS One, 1371; 8(7):2013.

15. World Health Organization: Global Tuberculosis Control Surveillance, Planning, Financing. Geneva, Swizerland: World Health Organization; 2005.(WHO/HTM/TB/2005.349), www.who.int/bookorders/anglais/detart1.jsp?s esslan, accessed febraury 2014

16. Tekle B, Mariam DH, Ali A. Defaulting from DOTS and its determinants in three districts of Arsi Zone in Ethiopia. Int J Tuberc Lung Dis, 2002; 6(7):573-579.

17. Baussano I, Pivetta E, Vizzini L, Abbona F, Bugiani M. Predicting tuberculosis treatment outcome in a low-incidence area. Int $J$ Tuberc Lung Dis, 2008; 12(12):1441-1448. 